\title{
Transition from multiple port to single port video-assisted thoracoscopic anatomic pulmonary resection: early experience and comparison of perioperative outcomes
}

\author{
Daniel G. French ${ }^{1}$, Calvin Thompson ${ }^{2}$, Sebastien Gilbert ${ }^{1}$ \\ ${ }^{1}$ Division of Thoracic Surgery, ${ }^{2}$ Department of Anesthesia, University of Ottawa, The Ottawa Hospital, 501 Smyth Road, Ottawa, ON, K1H 8L6, \\ Canada \\ Correspondence to: Sebastien Gilbert, MD, Associate Professor of Surgery, Chief. Division of Thoracic Surgery, The Ottawa Hospital, General \\ Campus Suite 6363, 501 Smyth Road, Ottawa, ON, K1H 8L6, Canada. Email: sgilbert@toh.on.ca.
}

\begin{abstract}
Background: Single port thoracoscopy is an approach aimed at minimizing trauma to the chest wall during lung resection. The objectives of this study were to describe early experience in the transition from multiple port video-assisted thoracic surgery (VATS) to single port surgery (S-VATS) and to compare perioperative outcomes between approaches.

Methods: Consecutive anatomic lung resections using S-VATS were reviewed and compared to a historical, prospective cohort of multiple port VATS cases. Outcomes analysis was focused on the use of operating room resources and postoperative recovery.

Results: Over 12 months, 50 S-VATS procedures were completed by one surgeon and compared to an equal number of VATS patients. The groups were similar in age, gender, BMI, comorbidity, tumor size and pulmonary function. There was no statistically significant difference in operative time. All tumors were completely resected (R0) and the median number of lymph nodes evaluated pathologically was equivalent $\{\mathrm{S}-\mathrm{VATS}=7$ [4-10]; VATS=7 [4-10]; $\mathrm{P}=0.92\}$. There was no significant difference in conversion rate $\{\mathrm{S}-\mathrm{VATS}=2(4 \%) ;$ VATS=1 $(2 \%) ; \mathrm{P}=0.56\}$. The median length of stay was similar in both groups $\{\mathrm{S}-\mathrm{VATS}=4$ [3-7]; VATS=4 [3-7]; $\mathrm{P}=0.99\}$. There was no mortality and no significant difference in the rate of major complications $\{\mathrm{S}-\mathrm{VATS}=10 / 50$ [20\%]; VATS=5/50 [10\%]; P=0.26\}. There was no difference in patient reported pain as measured by a visual analog scale at 24 hours $\{\mathrm{S}-\mathrm{VATS}=4$ [2-5]; VATS=4 [3-5]; P=0.63\}.

Conclusions: Early experience in the transition from multiple port VATS to S-VATS lung resection indicates that safety, efficiency and surgical quality are preserved. More long-term data are required. Alternative approaches to perform thoracoscopic lung resection should be carefully evaluated and compared to established minimally invasive techniques.
\end{abstract}

Keywords: Single port; uniportal surgery; thoracoscopy; video-assisted thoracic surgery (VATS); lobectomy; minimally invasive; perioperative outcomes

Submitted Nov 09, 2015. Accepted for publication Jan 21, 2016.

doi: $10.21037 /$ acs.2016.03.03

View this article at: http://dx.doi.org/10.21037/acs.2016.03.03

\section{Introduction}

Surgical instrumentation of the intercostal spaces and torque applied to ribs and costovertebral joints are major contributors to postoperative pain after thoracoscopic surgery. New operative approaches, such as single port thoracoscopy, have been developed with the intent to minimize trauma to these chest wall structures. Since the first report of single port pulmonary resection, the approach has been continually refined to allow safe completion of increasingly complex pulmonary resections (1-2). The objectives of this study were to characterize the transition from multiple port video-assisted thoracic surgery (VATS) 
to single port VATS anatomic lung resection (S-VATS) and to evaluate its impact on intraoperative and early postoperative outcomes. We had two hypotheses: (I) transition from VATS to S-VATS is safe and feasible for thoracic surgeons with experience in minimally invasive anatomic pulmonary resection and (II) S-VATS is associated with decreased early postoperative pain compared to VATS.

\section{Methods and materials}

\section{Patients and outcomes}

With approval from the Research Ethics Board, consecutive anatomic lung resections performed from August 2014 to August 2015 using S-VATS were retrospectively reviewed and compared to an equal number of multiple port VATS patients entered in a prospective database from December 2012 to May 2014. All operations were performed by a single surgeon. Baseline preoperative workup included a combined PET-CT scan and pulmonary function tests. Cerebral MRI was performed selectively for patients with larger tumors $(\geq 5 \mathrm{~cm})$, suspected stage IIIA nonsmall cell lung cancer (NSCLC), neurologic symptoms at presentation or limited pulmonary function. Patients aged over 70 years old with one or more risk factors for coronary artery disease had preoperative cardiac stress testing and/or cardiorespiratory exercise testing. Patients with a peripheral tumor $3 \mathrm{~cm}$ or less in size, a negative mediastinum on PET-CT and acceptable pulmonary function (FEV1\% and DLCO $\%>50 \%$ predicted) underwent mediastinal staging at the time of pulmonary resection.

Patient age, gender, body mass index (BMI), past history of smoking, Charlson comorbidity index and pulmonary function test results were recorded. Intraoperative outcomes included: tumor size, total number of lymph nodes sampled, surgical margin status, conversion to thoracotomy, number of stapler cartridges, number of chest tubes and operative time. Duration of chest tube drainage, postoperative pain assessment and length of stay were also recorded. Complications, both intraoperative and postoperative, were documented prospectively throughout the study period using our Thoracic Morbidity and Mortality (TMM) classification system (3), which is compatible with the Society of Thoracic Surgeons database. Each complication was assigned a grade according to methodology described in detail elsewhere (https://ottawatmm.org). In brief, grade I complications do not require an intervention, grade II complications require a medical intervention, grade III complications require a procedural intervention and grade IV complications are defined as organ failure requiring support available only in an intensive care unit. Postoperative pain assessment included the number of days on a patient controlled analgesia (PCA) device, the total dose of narcotic administered through the PCA reported as milligrams of hydromorphone, the level of pain reported by the patient using a visual analogue scale (VAS) (0 to 10) at 1 and 24 hours postoperatively and the patient satisfaction rating of pain control at 24 hours as a binary outcome $(0=$ not satisfied, $1=$ satisfied).

\section{Statistical analysis}

Categorical variables were analyzed for statistical significance using Fisher's exact test. Numerical variables were compared using the student's $t$-test or the Wilcoxon rank sum wherever appropriate. A P value less than 0.05 was considered significant and all tests of significance were twosided. Numerical continuous data is expressed as a median value with a $25^{\text {th }}-75^{\text {th }}$ percentile interquartile range (IQR). Statistical calculations were performed using SPSS 22 (IBM, Armonk, NY, USA).

\section{Single port technique}

The surgeon involved in the transition received formal training in advanced minimally invasive thoracic surgery and had over 10 years of experience with VATS anatomic lung resections. The established plan for transition to S-VATS was to enforce a low threshold for conversion to VATS should any compromise in exposure or safety arise during the operation. Guidelines for conversion to open thoracotomy were similar to VATS cases and included unexpected oncologic findings and/or hemorrhage that cannot be controlled using thoracoscopy. Finally, given our institutional commitment to the future of thoracic surgery, the transition would not be allowed to unfold at the detriment of the procedural experience and training of surgical residents.

Under general anesthesia and single-lung ventilation, the S-VATS anatomic resection was started by making a 3-5 $\mathrm{cm}$ access incision centered between the mid and anterior axillary lines in the fifth intercostal space. The incision for S-VATS was placed lower (fifth intercostal space) than the access incision used during multiple port cases (fourth intercostal space). A wound protector was inserted into the single access incision. A 30-degree, 10 


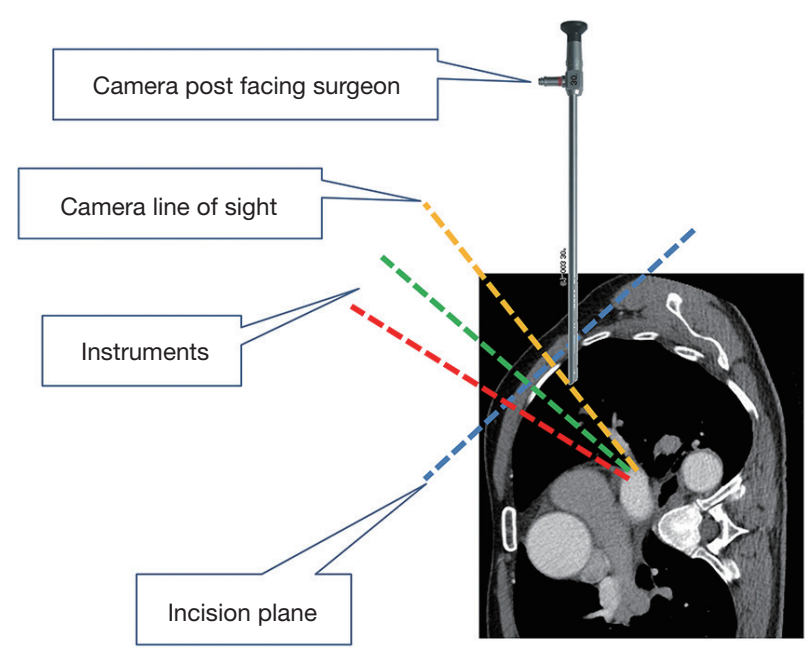

Figure 1 Relative position of the camera and surgical instruments within the single incision for anterior hilar dissection.

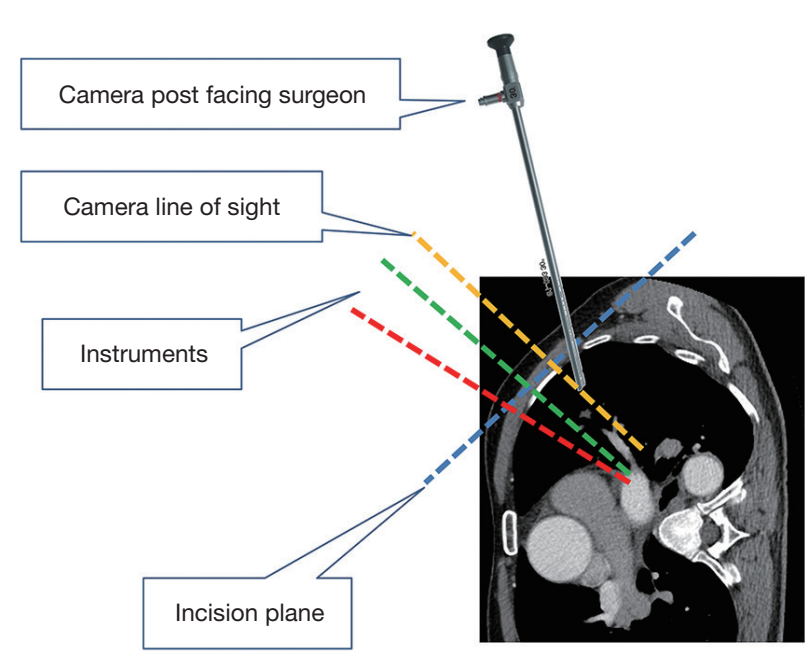

Figure 2 Camera position for anterior hilar dissection.

$\mathrm{mm}$ thoracoscope was placed at the posterior aspect of the wound and held in place using a supportive, flexible arm secured to the operating table. Figure 1 illustrates the optimal position of the scope, as well as the retracting and dissecting instruments within the intercostal space. Note is made of the change in position of the light source connector (i.e., camera post) in relationship to the camera head depending on whether dissection is occurring at the anterior or posterior hilum. As opposed to previous technical descriptions of S-VATS procedures, a 30-degree scope was selected in order to minimize potential conflicts

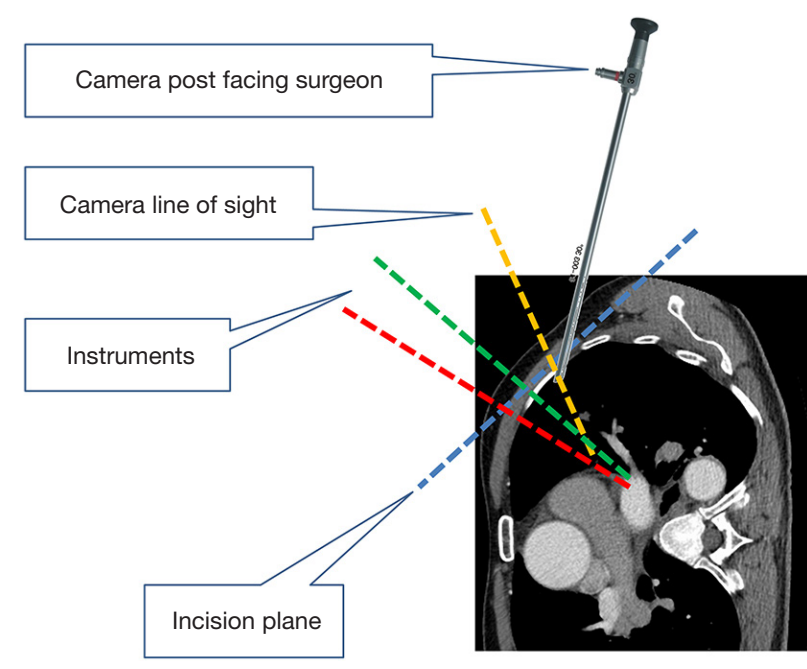

Figure 3 Camera leaning away from operating surgeon with 30-degree up orientation enhances view of anterior hilar structures.

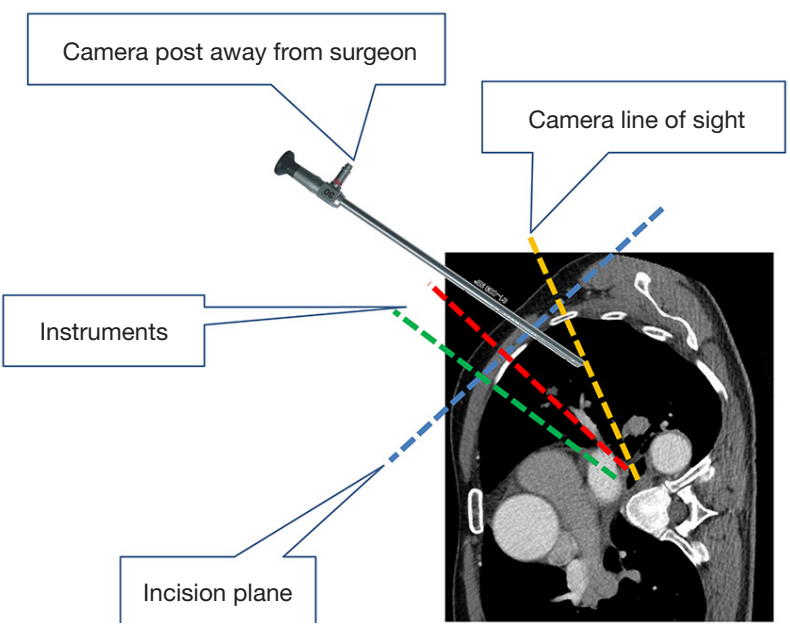

Figure 4 Camera leaning toward operating surgeon with 30-degree up orientation provides view of posterior hilar structures.

between the scope and surgical instruments. The monitor was placed on a straight line between the operating surgeon, camera and pulmonary hilum. Figures 2-5 illustrate how the angle of insertion of the camera and the position of the camera post (i.e., 30-degrees up or down) can be adjusted to allow optimal, circumferential visualization of the pulmonary hilum and mediastinum. All of the S-VATS procedures were performed using widely available, open surgical instruments with occasional use of laparoscopic alligator graspers. The operative setup requires minimal input from the surgical assistant since the surgeon can 

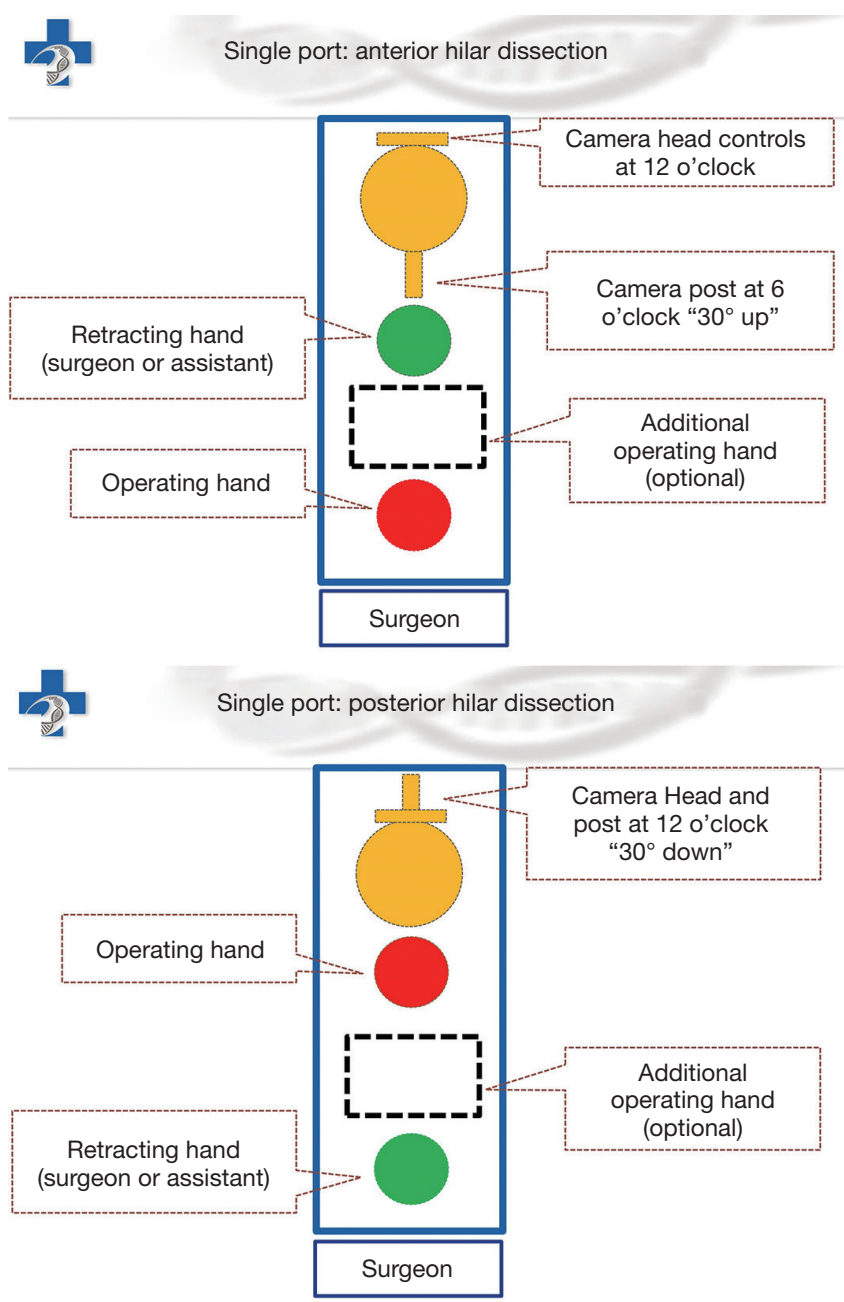

Figure 5 Camera leaning toward operating surgeon with 30-degree down orientation enhances view of posterior hilar structures and facilitates mediastinal lymph node sampling or dissection.

apply appropriate traction, expose, dissect and divide bronchopulmonary structures and pulmonary vessels. In addition, the surgeon can easily reposition the camera that is held steady by the scope stabilizer. With the exception of lower lobe segmental resections, dissection within the major fissure was avoided. As recommended for S-VATS resection of upper lobe tumors, the most proximal pulmonary arterial branch was divided first in order to allow safe division of the venous tributaries with the vascular stapler. This is not typically performed during multiple port VATS upper lobectomy. Another distinction is that, in S-VATS cases, the pleural space was drained with only one chest tube placed through the single port incision. All multiple port cases were drained with two chest tubes.

\section{Multiple port technique}

Multiple port VATS resection has been previously defined and described extensively (4). Briefly, a $10 \mathrm{~mm}$ port is placed at the eighth intercostal space at the mid-axillary line for the thoracoscope. The $3-5 \mathrm{~cm}$ access port was placed in the fourth intercostal space and centered over the anterior axillary line. A $5 \mathrm{~mm}$ port was placed in the auscultatory triangle along with another $5 \mathrm{~mm}$ port 2-3 interspaces below to allow assistance with both hands. In some cases, an additional anterior 5 or $10 \mathrm{~mm}$ port incision was made inferior to the access incision. In this approach, the camera is at an approximate 90-degree angle to the axis formed by the operating surgeon, hilar structures and video monitor.

\section{Results}

Patients in both the S-VATS and VATS groups were similar with respect to age, gender, BMI, smoking, comorbidity and pulmonary function tests (see Table 1). Most patients in both operative groups underwent resection of a primary NSCLC $\{$ S-VATS 48/50 [96\%]; VATS $=46 / 50$ [92\%]; $\mathrm{P}=0.68\}$. The type of resections performed and pathologic staging were also similar between the two groups as shown in Table 1. There was no significant difference in median tumor size $\{\mathrm{S}-\mathrm{VATS}=2.8 \mathrm{~cm}[\mathrm{IQR}=1.8-3.8]$; VATS $=2.5 \mathrm{~cm}[\mathrm{IQR}=1.6-3.5] ; \mathrm{P}=0.33\}$. All VATS and S-VATS surgical specimens had negative resection margins (R0) and contained an equivalent median number of lymph nodes available for pathologic analysis $\{\mathrm{S}-\mathrm{VATS}=7$ [IQR=4-10]; VATS=7 [IQR=4-10]; $\mathrm{P}=0.93\}$. Conversion rate to thoracotomy was low in both groups $\{\mathrm{S}-\mathrm{VATS}=2 / 50$ [4\%]; VATS $=1 / 50$ [2\%]; P=0.56\}. One VATS $(2 \%)$ and 1 S-VATS $(2 \%)$ procedure were converted to thoracotomy for oncologic reasons and 1 S-VATS (2\%) procedure was converted to thoracotomy for bleeding. One morbidly obese S-VATS patient (2\%) required the insertion of an additional port to facilitate use of the endostapler. There was no significant difference in the median number of stapler cartridges used per case $\{\mathrm{S}-\mathrm{VATS}=9[\mathrm{IQR}=8-10]$; $\mathrm{VATS}=8[\mathrm{IQR}=6-10] ; \mathrm{P}=0.58\}$. Median operative time was also statistically equivalent $\{$ S-VATS $=154$ minutes [142-175]; VATS=146 minutes [IQR=121-178]; $\mathrm{P}=0.08\}$. Two chest tubes were routinely used in the VATS group while only one chest tube was used in the S-VATS group. This did not translate into a significant difference in median duration of chest tube drainage $\{\mathrm{S}-\mathrm{VATS}=3$ days $[\mathrm{IQR}=3-5]$; VATS $=3$ days $[\mathrm{IQR}=2-5] ; \mathrm{P}=0.39\}$. Median length of stay 


\begin{tabular}{|c|c|c|c|}
\hline Parameter & VATS $(n=50)$ & S-VATS $(n=50)$ & $P$ value \\
\hline \multicolumn{4}{|l|}{ Patients } \\
\hline Age & $67[60-74]$ & 67 [64-73] & 0.82 \\
\hline BMI & 27 [24-30] & 27 [22-30] & 0.84 \\
\hline Charlson comorbidity index & $2[1-3]$ & $2[1-3]$ & 0.61 \\
\hline FEV1\% & 85 [69-101] & 79 [69-89] & 0.07 \\
\hline DLCO\% & 74 [63-85] & 73 [64-81] & 0.96 \\
\hline \multicolumn{4}{|l|}{ Operative details } \\
\hline Laterality (right:left) & $23: 27$ & $26: 24$ & 0.55 \\
\hline LLL & $6(12 \%)$ & $6(12 \%)$ & \\
\hline RLL & $5(10 \%)$ & $4(8 \%)$ & \\
\hline Other & $4(8 \%)$ & $1(2 \%)$ & \\
\hline Segmentectomy & $8(16 \%)$ & $10(20 \%)$ & 0.60 \\
\hline Operative time (minutes) & $146[121-178]$ & $154[142-175]$ & 0.08 \\
\hline Stapler cartridges & $8[6-10]$ & $9[8-10]$ & 0.58 \\
\hline Number of chest tubes & 2 [2-2] & $1[1-1]$ & $<0.01$ \\
\hline Conversion to open & $1(2 \%)$ & $2(4 \%)$ & 0.56 \\
\hline II & $5(10 \%)$ & $10(20 \%)$ & \\
\hline III & $1(2 \%)$ & $0(0 \%)$ & \\
\hline
\end{tabular}

${ }^{\dagger}$, All values reported are medians with interquartile range in parentheses unless otherwise specified; FEV1\%, percent predicted forced expiratory volume in 1 second; DLCO\%, percent predicted diffusion capacity for carbon monoxide.

was similar in both groups at 4 days $\{\mathrm{S}-\mathrm{VATS}=4$ days [IQR=3-7]; VATS=4 days [IQR=3-7]; $\mathrm{P}=0.99$ (Table 2).

There was no postoperative mortality in the study population. There was no significant difference in the number of patients who experienced a minor complication (TMM grade I-II) (S-VATS=9/50 (18\%); VATS $=13 / 50$ $(26 \%) ; \mathrm{P}=0.47)$. Major complication rates (TMM grade III-IV) were also similar between groups (S-VATS $=10 / 50$ [20\%]; VATS=5/50 [10\%]; P=0.26). Most of these were pleural complications which were resolved after insertion of a chest tube (S-VATS=6/50 (12\%); VATS=4/50 (8\%); $\mathrm{P}=0.74)$. Two other patients in the S-VATS group (4\%) required endoscopic procedures for hemoptysis and gastrointestinal bleeding. The grade IV complications were due to respiratory distress requiring transfer to intensive care for ventilatory support (S-VATS=2/50 (4\%); VATS=1/50 (2\%); $\mathrm{P}=1.0)$ (Table 2).

Five patients (10\%) in both the VATS and S-VATS groups had an epidural placed at the discretion of the anesthetist. The median number of days with a PCA was not 


\begin{tabular}{llll}
\multicolumn{2}{l}{ Table 2 Postoperative outcomes ${ }^{\dagger}$} & \\
\hline Parameter & VATS $(\mathrm{n}=50)$ & S-VATS $(\mathrm{n}=50)$ & P value \\
\hline Duration of chest tube drainage (days) & $3[3-5]$ & $3[2-5]$ & 0.39 \\
\hline LOS (days) & $4[3-7]$ & $4[3-7]$ & 0.99 \\
\hline Pain control & & \\
Duration of PCA (days) & $3[3-3]$ & $3[2-3]$ & 0.56 \\
Total PCA dose (mg) & $3.4(2.6-8.0)$ & $5.4(1.6-8.0)$ & 0.21 \\
VAS (1 hour) & $3[0-4]$ & $1[0-3]$ & 0.01 \\
VAS (24 hours) & $4[2-5]$ & $4[3-5]$ & 0.62 \\
Satisfied with pain control at 24 hours & $48(96 \%)$ & $47(94 \%)$ & 0.74 \\
Complications ${ }^{\ddagger}$ & & & \\
None & $32(64 \%)$ & $30(60 \%)$ & 0.68 \\
Minor (grade I-II) & $13(26 \%)$ & $9(18 \%)$ & 0.33 \\
Grade III & $4(8 \%)$ & $8(16 \%)$ & 0.22 \\
Grade IV & $1(2 \%)$ & $2(4 \%)$ & 0.56 \\
\hline${ }^{\dagger}$ All values reported are median and interquartile range, unless otherwise specified. LOS, length of stay; PCA, patient controlled \\
analgesia; VAS, visual analogue scale for pain; ${ }^{\ddagger}$ complications are graded according to the TMM system [(3), https://ottawatmm.org].
\end{tabular}

significantly different $\{\mathrm{S}-\mathrm{VATS}=3$ days $[\mathrm{IQR}=3-5]$; VATS $=3$ days $[\mathrm{IQR}=2-5] ; \mathrm{P}=0.39$ ]. There was no significant difference in the median dose of hydromorphone $\{\mathrm{S}-\mathrm{VATS}=5.4 \mathrm{mg}$ [IQR=1.6-8.0]; VATS=3.4 mg [IQR=2.6-8.0]; $\mathrm{P}=0.21$. There was a significant difference in the median VAS for pain at 1 hour postoperatively in favor of the S-VATS approach $\{\mathrm{S}-\mathrm{VATS}=1[\mathrm{IQR}=0-3]$; VATS $=3[\mathrm{IQR}=0-4]$; $\mathrm{P}<0.01\}$. However, at 24 hours the VAS in each group was similar $\{\mathrm{S}-\mathrm{VATS}=4[\mathrm{IQR}=3-5]$; VATS $=4[\mathrm{IQR}=2-5] ; \mathrm{P}=0.62\}$ (Table 2). There was no significant difference in the number of patients reporting satisfactory pain control at 24 hours $\{\mathrm{S}-\mathrm{VATS}=47 / 50[94 \%] ; \mathrm{VATS}=48 / 50[96 \%] ; \mathrm{P}=0.74\}$.

\section{Discussion}

The single port VATS approach to pulmonary resection has been under development for over 10 years (1). To our knowledge, the world-wide experience published in the English scientific literature remains limited with less than 1,000 cases of anatomic lung resection (2,5-12). Our general approach to the introduction of new surgical techniques or technology into clinical practice has emphasized safety and efficacy by carefully recording and analyzing outcomes $(13,14)$. It is only very recently that other comparative studies of multiple port and single port lung resection have started to emerge. Investigators from Asia have demonstrated that single port lung resection is safe when compared to the multiple port approach (10). Similarly, our results show comparable intraoperative and early postoperative outcomes. For the past several years, all intraoperative and postoperative adverse events occurring within our thoracic unit have been recorded prospectively and reviewed at weekly conferences. We have found that this methodology leads to more accurate and generally higher complication rates than retrospective review with partial or even complete patient sampling (15). Since all major procedures performed within out unit are submitted to the same scrutiny, the absence of any significant difference in complication grade or rate between S-VATS and VATS lung resections is a reliable indicator that patient safety was preserved during the transition period.

We also had concerns with regard to the impact of adopting a new surgical approach on the operative experience of surgical trainees. Although we do not yet have conclusive evidence, we observed that single port exposure, dissection and division of anatomic structures could be learned by thoracic surgery residents through closely supervised involvement in all parts of the procedure. At the beginning of our experience, we expected the learning curve and limited intrathoracic access to negatively impact operative efficiency. However, the results suggest otherwise as operating time for single port procedures was not significantly longer.

We thought that it would be valuable to describe the subjective experience associated with adopting this new 
surgical approach. We recognize that the lack of objective metrics to quantify this process is problematic. Nevertheless, there may be value in identifying successful strategies and pitfalls. The literature emphasizes the need for a gradual transition from multiple port to dual port to single port surgery (2). After reviewing the literature, attending lectures and courses and discussing specific technical aspects with experts, a decision was made to proceed directly from 4-5 ports to 1 port. We elected to apply the same patient selection criteria for S-VATS and VATS in order to ensure that the case volume would be sufficient to allow a steady climb up the initial part of the learning curve. As a matter of patient safety, the planned threshold for conversion from single port to multiple port VATS was low. We were initially concerned about the unavailability of dedicated S-VATS instruments at our institution. Although the potential advantages of special purpose instrumentation are undeniable, it is clear from our results that the single port approach also lends itself to the use of conventional instruments without compromising exposure or safety. We also elected to use a 30-degree instead of a 0 -degree thoracoscope because of familiarity with the instrument, availability of additional viewing angles and the option to create more room between the optics and the surgical instruments. Having gained experience with the single port approach, we agree with the argument that keeping the geometric plane of the scope as close as possible to the plane of the instruments produces a view of the surgical field that more closely resembles the open thoracotomy perspective when the surgeon is facing the patient's anterior chest (16).

There may be as many as $47 \%$ of patients reporting chronic pain symptoms after VATS procedures (17). It has been suggested that perioperative pain is decreased in patients undergoing a single port wedge resection or pleurectomy compared to patients having the same procedure using a multiple port technique (18). Other authors have claimed that accessing a single intercostal space minimizes postoperative pain but objective data to support this assertion is still lacking $(2,19)$. We can think of at least two potential explanations for decreased postoperative pain following S-VATS. First, only one anterior intercostal space is subjected to surgical injury. Secondly, positioning of the camera and instruments in a plane that closely parallels the intercostal space greatly reduces any torque forces that are commonly applied to the rib periosteum and intercostal nerve bundle during conventional thoracoscopy. We observed significantly lower VAS scores at 1 hour in the S-VATS group. Although this could be related to the approach per se, it could also be attributed to the fact that successful local anesthesia of a single interspace can probably be achieved more easily and reliably. Despite these advantages, the results did not show any differences in intraoperative narcotic use, postoperative narcotic use or pain scores at 24 hours. Clearly, more longterm objective data is needed before drawing conclusions. In our opinion, single port lung resection still represents a significant advance in minimally invasive thoracic surgery in terms of ergonomics and minimizing chest wall trauma. This approach has the potential to improve the currently limited adoption of the VATS approach for major lung resections $(20,21)$. As technology and techniques continue to evolve, we also look forward to the emergence of competing approaches that allow these procedures to be performed without any intercostal access (22).

In light of the study design and despite some degree of prospective data collection, the results are susceptible to potential biases inherent to retrospective reviews. We realize that generalizability is limited because the results reflect a single surgeon's experience. In addition, there are potential confounding factors which may have obscured the relationship between approach and postoperative pain. These include the routine use of pre-emptive, multi-level intercostal blocks which provide pain relief for several hours after surgery and the subjective differences in the individual patient's reaction to postoperative nociceptive stimuli. We acknowledge that any potential reduction in postoperative pain as a result of the single port approach could have become apparent later in the postoperative recovery and was thus undetectable using our methodology. Although we purposefully focused this report on intra-operative and early postoperative outcomes, we realize the importance of continued efforts to gather long-term data in order to provide a more complete picture of the potential impact of the single port approach on clinical outcomes.

Based on our early experience, transition from VATS to S-VATS anatomic lung resection can be accomplished while preserving intraoperative patient safety, oncologic quality and operating room resource utilization. Mid to long term pain outcomes will need further objective evaluation. More comparative data is needed to enable surgeons to critically appraise the potential benefits and drawbacks of alternative surgical approaches over established minimally invasive techniques.

\section{Acknowledgements}

The authors would like to express their deepest gratitude 
to our thoracic surgery care facilitator, Kristin Waddell $\mathrm{RN}$, and to all the members of our surgical team for their outstanding work, dedication, patience and support which were essential to making this transition a success.

\section{Footnote}

Conflicts of Interest: The authors have no conflicts of interest to declare.

\section{References}

1. Rocco G, Martin-Ucar A, Passera E. Uniportal VATS wedge pulmonary resections. Ann Thorac Surg 2004;77:726-8.

2. Gonzalez D, de la Torre M, Paradela M, et al. Videoassisted thoracic surgery lobectomy: 3-year initial experience with 200 cases. Eur J Cardiothorac Surg 2011;40:e21-8.

3. Seely AJ, Ivanovic J, Threader J, et al. Systematic classification of morbidity and mortality after thoracic surgery. Ann Thorac Surg 2010;90:936-42; discussion 942.

4. Swanson SJ, Herndon JE 2nd, D'Amico TA, et al. Videoassisted thoracic surgery lobectomy: report of CALGB 39802--a prospective, multi-institution feasibility study. J Clin Oncol 2007;25:4993-7.

5. Gonzalez D, Delgado M, Paradela M, et al. Uni-incisional video-assisted thoracoscopic left lower lobectomy in a patient with an incomplete fissure. Innovations (Phila) 2011;6:45-7.

6. Gonzalez-Rivas D, Paradela M, Fieira E, et al. Singleincision video-assisted thoracoscopic lobectomy: initial results. J Thorac Cardiovasc Surg 2012;143:745-7.

7. Gonzalez-Rivas D, Paradela M, Fernandez R, et al. Uniportal video-assisted thoracoscopic lobectomy: two years of experience. Ann Thorac Surg 2013;95:426-32.

8. Gonzalez-Rivas D, Fieira E, Delgado M, et al. Is uniportal thoracoscopic surgery a feasible approach for advanced stages of non-small cell lung cancer? J Thorac Dis 2014;6:641-8.

9. Anile M, Diso D, Mantovani S, et al. Uniportal video assisted thoracoscopic lobectomy: going directly from open surgery to a single port approach. J Thorac Dis 2014;6:S641-3.

10. Shen $Y$, Wang H, Feng M, et al. Single- versus multipleport thoracoscopic lobectomy for lung cancer: a propensity-matched study†. Eur J Cardiothorac Surg 2016;49 Suppl 1:i48-i53.

11. Chung JH, Choi YS, Cho JH, et al. Uniportal videoassisted thoracoscopic lobectomy: an alternative to conventional thoracoscopic lobectomy in lung cancer surgery? Interact Cardiovasc Thorac Surg 2015;20:813-9.

12. Hsu PK, Lin WC, Chang YC, et al. Multiinstitutional analysis of single-port video-assisted thoracoscopic anatomical resection for primary lung cancer. Ann Thorac Surg 2015;99:1739-44.

13. Gilbert S, McGuire AL, Maghera S, et al. Randomized trial of digital versus analog pleural drainage in patients with or without a pulmonary air leak after lung resection. J Thorac Cardiovasc Surg 2015;150:1243-9.

14. McGuire AL, Gilbert S. Transthoracic Extracorporeal Gastric Conduit Preparation for Minimally Invasive IvorLewis Esophagectomy. Innovations (Phila) 2015;10:23640; discussion 240.

15. Campbell PG, Malone J, Yadla S, et al. Comparison of ICD-9-based, retrospective, and prospective assessments of perioperative complications: assessment of accuracy in reporting. J Neurosurg Spine 2011;14:16-22.

16. Bertolaccini L, Rocco G, Viti A, et al. Geometrical characteristics of uniportal VATS. J Thorac Dis 2013;5 Suppl 3:S214-6.

17. Steegers MA, Snik DM, Verhagen AF, et al. Only half of the chronic pain after thoracic surgery shows a neuropathic component. J Pain 2008;9:955-61.

18. Jutley RS, Khalil MW, Rocco G. Uniportal vs standard three-port VATS technique for spontaneous pneumothorax: comparison of post-operative pain and residual paraesthesia. Eur J Cardiothorac Surg 2005;28:43-6.

19. Salati M, Brunelli A, Rocco G. Uniportal video-assisted thoracic surgery for diagnosis and treatment of intrathoracic conditions. Thorac Surg Clin. 2008;18:305-10, vii.

20. Boffa DJ, Allen MS, Grab JD, et al. Data from The Society of Thoracic Surgeons General Thoracic Surgery database: the surgical management of primary lung tumors. J Thorac Cardiovasc Surg 2008;135:247-54.

21. Begum S, Hansen HJ, Papagiannopoulos K. VATS anatomic lung resections-the European experience. J Thorac Dis 2014;6 Suppl 2:S203-10.

22. Liu CY, Lin CS, Liu CC. Subxiphoid single-incision thoracoscopic surgery for bilateral primary spontaneous pneumothorax. Wideochir Inne Tech Maloinwazyjne 2015;10:125-8.

Cite this article as: French DG, Thompson C, Gilbert S. Transition from multiple port to single port video-assisted thoracoscopic anatomic pulmonary resection: early experience and comparison of perioperative outcomes. Ann Cardiothorac Surg 2016;5(2):92-99. doi: 10.21037/acs.2016.03.03 